\title{
Development Of E-Health In Austria, Bulgaria, Croatia, Estonia And Slovakia
}

\author{
Angel Atanasov Georgiev \\ Faculty of Public Health, Medical University - Sofia, Bulgaria \\ Doroteya Shtereva-Tzouni \\ Faculty of Public Health, Medical University - Sofia, Bulgaria
}

\begin{abstract}
Electronic health services (e-Health) are now a fixed element of the health care system and cover a wide range of applications, such as the electronic health insurance card, the health record and an overview of medications, plus telemedicine. A prerequisite for this is the secure transmission of medical data for preventive medicine, diagnosis, treatment and the ongoing support of patients in the form of text, sound recordings and/or images. Uneven development of eHealth solutions within the EU27 remains a major obstacle in providing European citizens with a satisfying access to cross-border healthcare and the main challenge for the EU is harmonizing the systems. The slow shift of the paradigm centralizing the role of the patient will become more rapid making our assumptions about the savings thanks to the technology even more conservative than they are. Building on the trust that Europeans have in health institutions and the way they convey themselves is essential.
\end{abstract}

Keywords: e-health, m-health, health expenditure, Austria, Bulgaria, Croatia, Estonia, Slovakia

\section{INTRODUCTION}

Europe's countries have made substantial progress towards modern eHealth infrastructures and implementations, thereby leading the rest of the world. Following the Communication of the European Commission (EC) on "eHealth - making healthcare better for European citizens: An action plan for a European eHealth Area" [1], Member States of the European Union (EU) committed themselves "to develop a national or regional roadmap for e-Health.

Electronic health services (e-Health) are now a fixed element of the health care system and cover a wide range of applications, such as the electronic health insurance card, the health record and an overview of medications, plus telemedicine. A prerequisite for this is the secure transmission of medical data for preventive medicine, diagnosis, treatment and the ongoing support of patients in the form of text, sound recordings and/or images. e-Health offers not only technological opportunities, but also a chance in terms of structural policy to network the increasingly divided health services (in the sense of a division of labour) by means of information and communications technologies. 
It goes without saying that more and more people are using the internet to research their health conditions. This means not only looking up symptoms, but exploring treatment methods and medicines on the web. The Internet has empowered patients to make decisions about their next steps. Nurses and doctors use hand-held computers to update a patient's medical record and check whether they are administering the correct treatment. Results of lab tests, records of vital signs, and medicine orders are stored in a database that can be referred to later. And as more institutions adopt electronic health records, patients have easier access to their own information so they, too, can understand their treatment. With vast patient history, scientists can better study trends and causes of ailments. The advantages of the eHealth services could be summed up as: Improved quality of care; Better planning and resource allocation; Cost efficiency - more efficient health landscape; Enhancing the evidence base for health service delivery and policy making; Real-time monitoring; Providing better, tailored and personalized services; Preemptive measures [2].

\section{COMPARISONS}

$48 \%$ of people living in Europe say they have access to a web-based or a telephone healthcare information service that is publicly available in all parts of the country which, running $24 / 7$, and being interactive. ePrescriptions are still underdeveloped. $37 \%$ of Europeans say that ePrescription services are in use in their countries. One of the barriers in development of data usage in healthcare is the trust patients have to healthcare providers. Nearly three quarters of people $(74 \%)$ say they trust health and medical institutions to protect their personal information, with $24 \%$ of people fully trusting them, and $50 \%$ only tending to trust them (Eurobarometer 2015a). Therefore, there is a raising need for increasing data protection awareness and digital health literacy, especially like countries like Croatia where almost $1 / 3$ of the population does not trust the healthcare institutions.

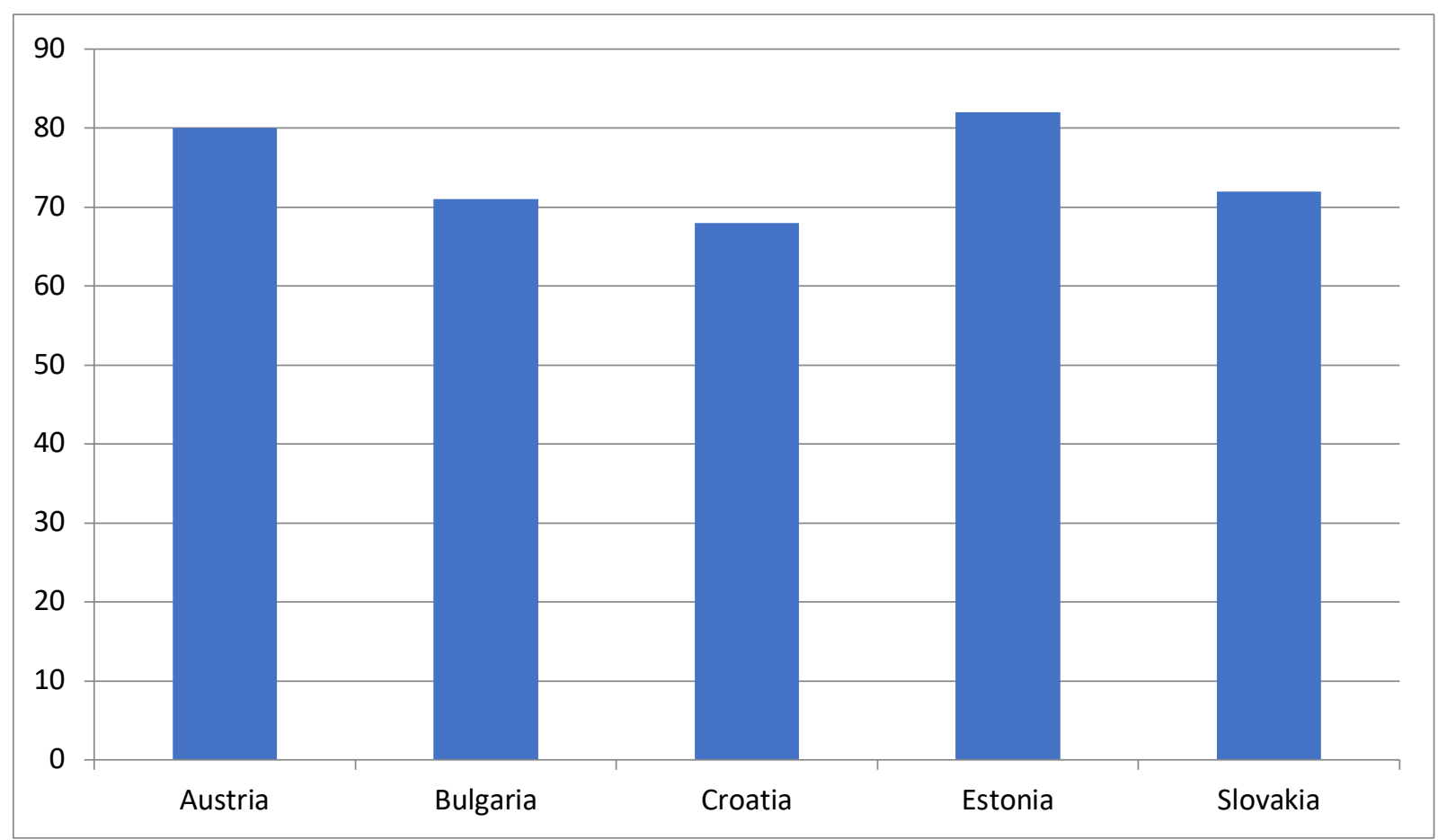

Fogire1: Percentage of respondents declaring they trust healthcare institutions managing their health data [3] 
In order to assess how well countries are performing with regard to development of eHealth services is compiled a index, which enables to see how the new technology is used in European countries (It is a summary measure of average achievement in five measures: availability of online appointment booking, e-Prescription status, 24/7 healthcare info service availability and usage of online appointments and usage of ePrescriptions by GPs. The indices were normalized and transformed from a raw variable into a unit-free index between 0 and 10 (which allows different indices to be added together). According to this index development of eHealth services in Bulgaria is very weak, and the country takes the last place in European union. Countries like Estonia manage to achieve significant success in the realization of eHealth and hold a leading position within the EU.

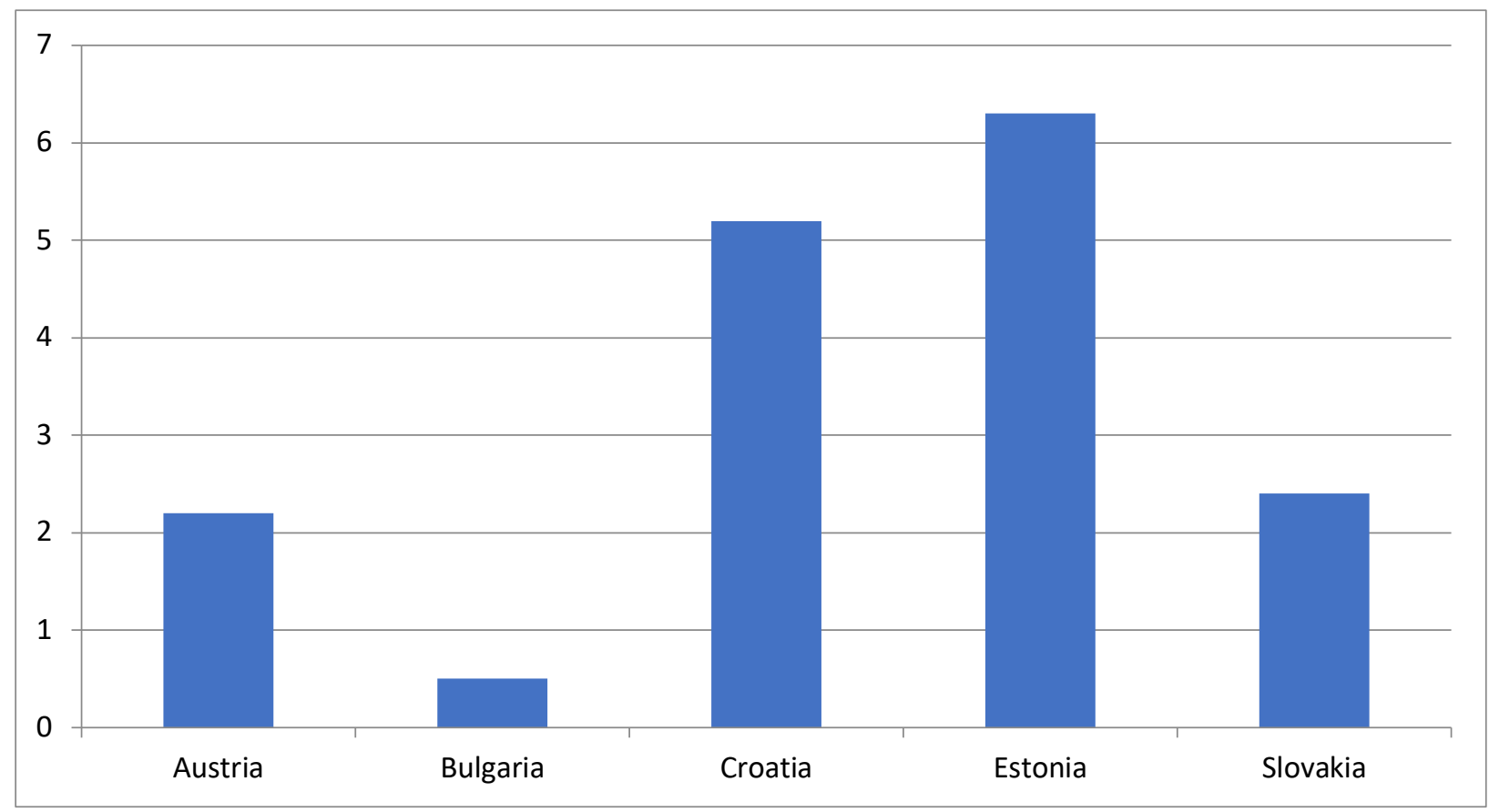

Figure 2: Ehealth index in points (2016) [4]

Healthcare costs continue to rise. Patients, clinicians, and policy makers are concerned whether it is possible to control costs while maintaining the quality of healthcare services. The use of information and communication technology (ICT) in healthcare sector (eHealth) could become a useful tool aimed at increasing efficiency, improving access, and raising the quality of care, especially in the case of much wasteful spending due to over usage of certain health services [5]. The implementation of eHealth systems is generally expensive and will have an impact on different healthcare providers, patients, and other stakeholders. Policy makers need demonstrable evidence of costs and benefits. If large-scale eHealth implementation warrants governmental investment, this will also require demonstrable benefits for the patients, providers, and society at large [6]. 
Georgiev, A. A., \& Shtereva-Tzouni, D. (2020) Development Of E-Health In Austria, Bulgaria, Croatia, Estonia And Slovakia. Archives of Business Research, 8(4). 155-162.

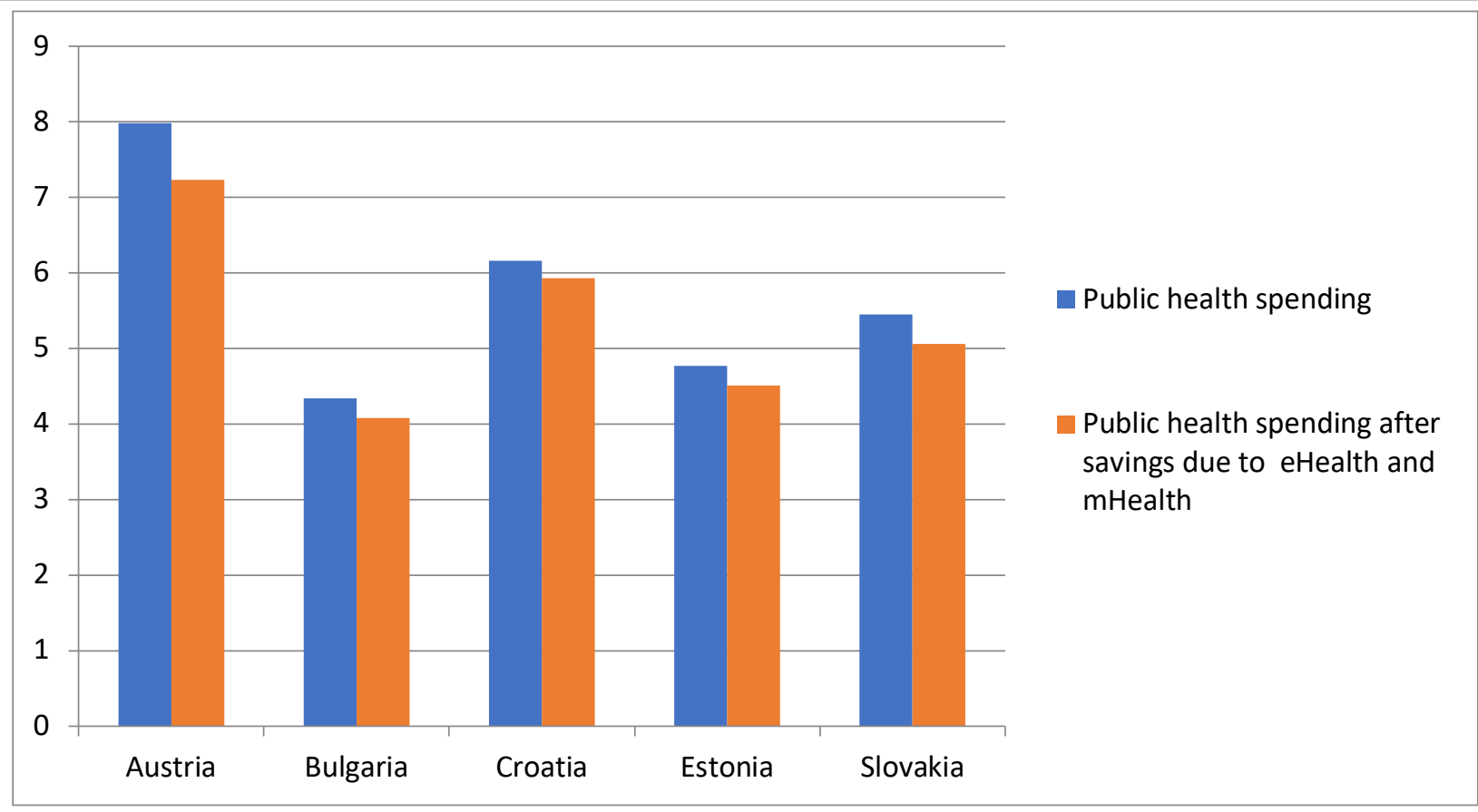

Figure 3: Health expenditures and possible savings thanks to eHealth and mHealth[7] (\% of GDP

\section{Austria}

[8])

As early as 2005, the introduction of the electronic health insurance card (e-Card) for all persons covered by health insurance - alongside the administrative simplification of using cashless health care services - represented an important condition for the Austrian Electronic Health Record (ELGA). Currently the e-Card is being further developed in a technological sense as well as with regard to safety - the latter will also be improved by the inclusion of a photo of the holder.

The Electronic Health Record (ELGA) is an information system which provides patients and the doctors, hospitals, care facilities and pharmacies treating them with easy access to certain health data from anywhere and at any time. This improves the flow of information, supports the medical, therapeutic and nursing support of patients, and increases patient safety. ELGA contains medical and nursing discharge letters from hospitals, laboratory and radiological results as well as information about prescribed and dispensed medication. The data of a person remains in the place where it originated, but ELGA networks this data and makes it available electronically via a link.

The entire population participates in ELGA, unless they have opted out of it, as do the legally established ELGA health services providers. Everyone has at any time a right to inspect and access their own ELGA health data. Health service providers have access to patients who are currently being treated or supported by them (usually for 28 days; however, patients have the right to individually determine access to and the duration of access to their ELGA health data). Pharmacies have access to the e-Medication list of a patient, but only for two hours. Patients who have not opted out of ELGA can view the e-Medication list of medicines they have been prescribed and which they have (or have not yet) picked up from a pharmacy Via technical measures and also via legal provisions, ELGA also improves data protection for patients. 
Data transmission always takes place in encrypted form and within safe health networks which have been specially established for the health care system. Access to data in the technical area is only permitted on the basis of the dual control principle, or with comparable technical security measures. In addition, only authorised ELGA health services providers can access the data of their patients, and only as long as they are treating or supporting them. In the ELGA records, patients can at any time see who has accessed their health data, when they did so, and which data they have viewed. They also have the right to administrate their own health data, and the opportunity to wholly or partially opt out of ELGA, or to opt back in again [9].

The legal basis for ELGA was adopted at the end of 2012. Since the beginning of 2014, the ELGA portal (Link in German: ELGA-Portal, Information in English), die ELGA service line and the ELGA opting-out service have been in operation. Since the end of 2015, ELGA is being introduced in stages, starting with public hospitals. In 2016, a test run was carried out with the ELGA function eMedication. In the period March 2018 to September 2019, ELGA and e-Medication are being made available in stages to doctors in private practices, group practices, pharmacies and outpatient clinics. These will be followed by private hospitals, and subsequently by dentists with health insurance fund contracts. In addition, a pilot project for an e-Vaccination pass is being prepared.

In the framework of the eHealth strategies study, ePrescription is understood as the process of the electronic transfer of a prescription by a healthcare provider in a primary care or community health centre setting to a pharmacy for retrieval of the drug by the patient. A necessary condition for this to occur is the recording of medications in the prescriber's office Electronic Medical Record (EMR) or other system in order to generate an electronic document, the medication prescription, to be transferred via communications connections to a specific pharmacy or a regional or national ePrescription repository. The ePrescription process in primary care needs to be distinguished from the use of computer technology in hospitals to facilitate the medication prescription and administration process. In those types of settings, the gold standard is a closed loop medication administration system which may include medication reconciliation and adverse drug event monitoring. Closed loop medication systems usually include an electronic medication administration record (eMAR) as well as the use of Computerized Provider/Physician Order Entry (CPOE) by physicians and/or other clinicians and support staff [10].

\section{Estonia}

The Estonian e-health system is among the world's most ambitious and a clear example of why this small EU country is widely hailed as one of the most advanced digital nations in Europe. Already, more than $95 \%$ of the data generated by hospitals and doctors has been digitised, and citizens can enjoy easy access to their own medical records, prescriptions, and the most suitable health professional. This system - which not only improves the cost-effectiveness, sustainability and efficiency of the Estonian healthcare service but also facilitates the transition to preventive, rather than curative, medicine - is underpinned by blockchain technology, a crucial pillar in ensuring the integrity and security of all patient data.

Patients no longer need to spend valuable time seeing a doctor for a repeat prescription: they can just call their doctor and then head straight to the pharmacy to fill their digital prescription. There are more direct economic benefits with regard to printing costs of electronic prescriptions. In Estonia, printing costs for paper prescriptions went down from EUR 63,668 in 2009 to around EUR 
1,000 in 2010 (Parv et al. 2016). The breakeven point for the country's investment was nearly achieved by mere reduction of paper used: the paper forms, printing and storing them securelyso the cost of the system and the maintenance currently is cheaper than if they bought the paper prescriptions. A survey in Estonia also supported perceived time savings, with repeated prescriptions now taking about 10-15 seconds, and new prescriptions taking about 30-60 seconds (Deetjen 2016).

\section{Slovakia}

After nearly 10 years of waiting, Slovakia launched the eHealth system on January 1, 2018. It's expected to improve health care and quality of life. The system has cost $€ 80$ million so far. The launch of the system was protested by medical organisations that complained it was not prepared for all outpatient departments. With this system in place, patients only need to show doctors their ID cards. Before, they had to use health insurance cards. The doctors will scan the cards with the reader and will immediately receive the patients' medical information. The medical records will also be available online and doctors can prescribe medication online. Once patients receive their online prescriptions, they can show their ID card at the pharmacy where the online prescription will be accessible [11]. Health records of patients are stored in the National Health Information System (eHealth System), therefore a secured approval of the patient, as well as of the health professional who creates or accesses the health records is important. Health records in Electronic Health Book represent an indispensable tool for the health professionals in charge to successfully set diagnose and following treatment. Patient is informed about his health status online anytime [12].

\section{Croatia}

In Croatia, eHealth is a priority area of the eCroatia programme and includes the computerization of primary health services, the introduction of an Integrated Healthcare Information System and the computerization of the Croatian Institute for Health Insurance.

All health care providers, public and private, create and send data to the national registries and databases, or create reports on their activities. The primary data sources in this context are all health care providers in Croatia including GP practices, hospitals, health care centers, and private care providers. The recipients of the data are the Health insurance information system (for financial reimbursement and health insurance purposes), the Central health care information system (as the main data exchange system at a national level), and the National public health information system (a platform that hosts all public health registries, including surveillance and reporting services). As unique identifiers have been compulsory in Croatia for forty years for all citizens, all these information sources can also be indirectly or directly linked to national registries, databases, reports, and international sources where relevant. It is, therefore, possible to use these linked data to provide policy makers and other stakeholders with new and useful insights based on analyses of big health care data. It is apparent that routinely collected data in the Croatian health care system have become abundant and well supported by the information and communication technology. These health information systems have been successful in fulfilling their primary purpose, however, attempts at secondary use have been patchy.

\section{Bulgaria}

Unfortunately, the development of the healthcare system in Bulgaria is not satisfactory, Bulgaria still has no full e-health system, although it is a permanent priority in all government programs and 
national health strategies over the past decade (Strategy for implementation of electronic healthcare in Bulgaria, National Health Strategy 2014-2020, Government Programme for sustainable development of the Republic of Bulgaria 2015). The institutions have acted inefficiently and the resources for building the system have remained unused.

The benefits of E-Health in Bulgaria for patients should include: easier access, better quality, speed and convenience, awareness and participation in control; and for doctors: reducing the administrative burden, eliminating unnecessary documentation, timely and ease of access information for finding evidence-based solutions, easier communication, ways to plan activities more effectively.

An essential tool for ensuring the effective functioning of the health system is the integration and connectivity of healthcare by building a national health information system and ensuring public access to the system through an electronic identifier. A unified information system of health care is the basis on the development of which E-Health will be built with its main components: electronic health records, electronic prescription, electronic referral, electronic portal, etc. Its implementation allows for more online administrative and health services sector activities, providing access to information about the patient's own health, improving relations between different levels of the system, improving the quality of medical services and making the spending of public funds for health care more effective.

The main EU recommendations for including EHealth in Bulgaria are: Need of a well-established health strategy, as a precondition for access to the structural funds; Need of an action plan of this strategy before planning the investments needed; Adoption of EU standards for interoperability should guide the investments in E-Health; Continuous and strong involvement in EU initiatives [13].

\section{CONCLUSIONS}

Uneven development of eHealth solutions within the EU27 remains a major obstacle in providing European citizens with a satisfying access to cross-border healthcare and the main challenge for the EU is harmonizing the systems. The slow shift of the paradigm centralizing the role of the patient will become more rapid making our assumptions about the savings thanks to the technology even more conservative than they are. Building on the trust that Europeans have in health institutions and the way they convey themselves is essential. In Bulgaria, Croatia and Slovakia around $30 \%$ of the population does not trust the health institutions, which may be an obstacle to the development of eHealth. Data security and privacy are the key concerns which have been expressed by citizens. In particular, individuals as consumers of healthcare must have confidence that personal data concerning health will not be misused, will be stored correctly and high cybersecurity standards are applied for personal data concerning health. Effective national health reform needs to adopt the perspective of the patient in order to understand how eHealth tools and services can be used to facilitate better care and to ensure populations are more proactively involved in their own health and well-being.

EHealth provides an opportunity to significantly reduce health care costs as well as improve the efficiency, accessibility and quality of health services. Bulgaria is one of the countries in the EU where the introduction of eHealth is significantly slowing down while countries like Estonia are 
among the best examples of its implementation. The need for a timely introduction of eHealth in Bulgaria is particularly important in order to improve the efficiency of the health system, to achieve transparency and to eliminate corruption. Progress in national eHealth adoption is often influenced by a range of factors that extend beyond the obvious requirements of skills and funding for technology and it is here that intersectoral engagement of stakeholders, led by the health ministry, is a key catalyst for success.

\section{References}

1. European Commission. eHealth - making healthcare better for European citizens: An action plan for a European eHealth Area. Brussels: COM(2004)356 final.

2. Polityka Insight. Transforming eHealth into a political and economic advantage, 2017

3. Eurobarometer, 2015

4. Polityka Insight, 2017

5. OECD, 2017

6. Telemedicine Journal and e-Health. Bashshur R., 2013

7. MHealth is an abbreviation for mobile health; mHealth broadly encompasses the use of mobile telecommunication and multimedia technologies as they are integrated within increasingly mobile and wireless health care delivery systems.

8. Polityka Insight, 2017

9. Sozialministerium Austria https://www.sozialministerium.at/cms/siteEN/attachments/6/3/9/CH4129/CMS1417371984289/bmasgk_th e-austrian_health-care-system_keyfacts_web.pdf

10. http://ehealth-strategies.eu/report/eHealth_Strategies_Final_Report_Web.pdf

11. https://spectator.sme.sk/c/20729936/slovakia-launches-online-health-care.html

12. https://www.ezdravotnictvo.sk/en/electronic-health-book

13. Stanchev P. and Foteva E. Bulgarian E-Health Overview 2015

14. https://ec.europa.eu/digital-single-market/en/news/ehealth-adoption-primary-healthcare-eu-rise 